\title{
TIPE2-modified human amnion-derived mesenchymal stem cells promote the efficacy of allogeneic heart transplantation through inducing immune tolerance
}

\author{
Feng Wang ${ }^{1,2,3,4 \#}$, Guanping Yao ${ }^{5 \#}$, Sisi Pan ${ }^{1}$, Xin Mao ${ }^{6}$, Xu Zhao ${ }^{6}$, Chuntian $\mathrm{Li}^{6}$, Zheng Hong ${ }^{6}$, \\ Guiyou Liang ${ }^{1,4}$, Limei Yu ${ }^{5}$, Xuanyi Hu ${ }^{4}$, Wanfu Peng ${ }^{4}$
}

${ }^{1}$ Department of Clinical Medical College, Guizhou Medical University, Guiyang, China; ${ }^{2}$ Department of Cardiac Surgery, The Affiliated Hospital of Zunyi Medical University, Zunyi, China; ${ }^{3}$ Department of Cardiothoracic Surgery, The Second Affiliated Hospital of Zunyi Medical University, Zunyi, China; ${ }^{4}$ Department of Cardiac Surgery, The Affiliated Hospital of Guizhou Medical University, Guiyang, China; ${ }^{5}$ Department of Guizhou Regenerative Medicine Laboratory, The Affiliated Hospital of Zunyi Medical University, Zunyi, China; ${ }^{6}$ Department of Clinical Medical College, Zunyi Medical University, Zunyi, China

Contributions: (I) Conception and design: All authors; (II) Administrative support: F Wang, G Liang; (III) Provision of study materials or patients: F Wang, G Yao, X Mao, X Zhao, S Pan; (IV) Collection and assembly of data: F Wang, G Yao, X Mao, X Zhao, S Pan; (V) Data analysis and interpretation: F Wang, G Yao, X Mao, X Zhao, S Pan, X Hu; (VI) Manuscript writing: All authors; (VII) Final approval of manuscript: All authors.

\#These authors contributed equally to this work.

Correspondence to: Guiyou Liang. Department of Cardiac Surgery, The Affiliated Hospital of Guizhou Medical University, Guiyang 550000, China. Email: guiyou515@163.com.

Background: Immune rejection of heart transplantation has been regarded as the biggest challenge encountered by a patient suffering from end-stage heart disease. The transplantation of human amnionderived mesenchymal stem cells (hAD-MSCs) has exhibited promising application prospects in organ transplantation. However, its persistent unsatisfactory tolerance has limited the widespread application of this technology. We aim to investigate the role of tumor necrosis factor- $\alpha$-induced protein- 8 like- 2 (TIPE2)-mediated hAD-MSCs in immune tolerance in heart transplantation and its molecular regulatory mechanisms.

Methods: This project detected the effect of TIPE2 on immune tolerance by constructing an allogeneic heart transplantation mouse model through which TIPE2-overexpressed hAD-MSCs were injected into recipients. The fluorescence distribution of TIPE2-hAD-MSCs in mice was observed by a small animal in vivo imaging system. Pathological changes of the transplanted heart were detected by hematoxylin and eosin (HE) staining. Flow cytometry was performed to detect the content of cardiac lymphocytes. The expression of immuneinduced related factors was measured by quantitative real-time PCR (qRT-PCR) and western blot assays.

Results: TIPE2-hAD-MSCs protected myocardial tissue structures, reduced the spleen and thymus indexes in recipient mice, minimized the content of cardiac lymphocytes, reduced expressions of $E R K, p 38$, and $I F N-\gamma$, and elevated expressions of both $I L-10$ and $T G F-\beta$, markedly improving the survival time and survival rates of recipient mice.

Conclusions: TIPE2-hAD-MSCs induce immune tolerance and improve the survival rates of allogeneic heart transplantation in mice. This study is expected to offer an ideal source and target of cells for organ transplantation.

Keywords: Tumor necrosis factor- $\alpha$-induced protein-8 like-2 (TIPE2); human amnion-derived mesenchymal stem cells (hAD-MSCs); heart transplantation; immune tolerance

Submitted Jun 07, 2021. Accepted for publication Aug 05, 2021.

doi: $10.21037 /$ jtd-21-1034

View this article at: https://dx.doi.org/10.21037/jtd-21-1034 


\section{Introduction}

As an effective treatment approach for patients with end-stage heart disease, the major obstacles to heart transplantation are immunosuppression and myocardial ischemia reperfusion. To avoid the complications caused by longterm immunosuppression, inducing donor-specific immune tolerance is recognized as an optimized approach to suppress rejection. In recent years, mesenchymal stem cells (MSCs) have been explored in the disciplinary field. They are not only recognized as a type of adult stem cell with characteristics such as high self-replication, multidirectional differentiation, and promotion of cell implantation and hematopoietic support but are also considered as regulators of great potential, with characteristics of immune regulation (1). They can inhibit the occurrence of allogeneic graft versus host disease, induce immune tolerance, and prolong the survival time of the graft. In particular, the potential of human amnion-derived MSCs (hAD-MSCs) is more remarkable $(2,3)$. Their advantages include extensive sources, amplification in vitro, no ethical issues, and are convenient to obtain, among others. Moreover, they also manifest low immune rejection reactions, are able to self-replicate, have low immunogenicity, and have a strong involvement in immune regulation and damage reparability. They can be differentiated into specialized tissue cells under certain conditions. Furthermore, these cells possess the characteristics of migration to damaged tissues and inflammatory areas and homing (4-6). In general, their application holds promise in major diseases encompassing organ transplantation and ischemia-reperfusion injury. Research on immune tolerance and mechanisms has assisted the translation of fundamental research into clinical practice, thereby facilitating investigations into immune characteristics and regulatory mechanisms of hAD-MSCs. This provides important theoretical value and clinical significance in the treatment of major immune diseases.

Studies have proven that MSCs function as inhibitors of the proliferation of T cells, B cells, and NK cells, as well as DC maturation. Moreover, regulatory T cell (Treg) amplification can be induced both in vivo and in vitro, which inhibits the immune rejection reaction and prolongs the survival time of the allograft, thereby inducing immune tolerance. MSCs can either exert immune effects through direct contact with immune cells or are regulated by the secretion of soluble factors such as IDO, TGF- $\beta, P G E 2$, $H G F, I L-10, I F N-\gamma, N O$, and LIF (7-9). They either act alone or work synergistically. In recent years, research on hAD-MSCs has attracted increasing attention. Study results on immune tolerance have mainly focused on in vitro effects $(10,11)$. MSC supernatant in culture has been shown to inhibit $\mathrm{T}$ cell proliferation. Both $\mathrm{T}$ cell proliferation and apoptosis can be inhibited by cell contact. The expression levels of Fas-L and MIF have been detected at the RNA level, and one of the mechanisms might involve blocking the caspase- 3 pathway $(12,13)$. From inducing immune tolerance to diverse immune cells and the underlying mechanisms, there is much about hADMSCs to be investigated. However, priority has been given to the application prospects of amniotic stem cells, which underlie anti-rejection/ischemia-reperfusion injury in organ transplantation (6). In a report by Gorjipour et al., they investigated the efficacy of hAD-MSCs in treating chronic models of myocardial ischemia and heart failure in rats (14). They found that hAD-MSCs were effective in the early stages of myocardial ischemia and did not have a significant advantage in patients with chronic HF. We wondered whether genetic modification of hAD-MSCs could further improve their prospects for use in cardiac transplantation.

Tumor necrosis factor- $\alpha$-induced protein- 8 like- 2 (TIPE2) gene-modified hAD-MSCs were shown to facilitate the induction of more robust immune tolerance. TIPE2 is a family member of tumor necrosis factor $\alpha$-induced protein 8 (TNFAIP8) $(15,16)$. As a newly discovered important molecule, it functions as a negative regulator in balancing the body's immune system. During the culture of TIPE2 knockout T cells, cytokine IFN- $\gamma$ in Th1 cells and cytokine IL-4 in TH2 cells were significantly increased. Other cytokines CD25, CD44, and CD69 were also significantly increased. The immune responses of CD4+ and CD8+ T cells were significantly enhanced in TIPE2-/- rats compared to the control group. In response to stimulation by foreign antigens, bone marrow-derived TIPE2-/- macrophages produced more IL-6, IL-12 than wild type. TIPE2 plays an important negative regulatory role in the intrinsic and adaptive immune responses $(17,18)$. Our previous study findings demonstrated that TIPE2overexpressed hAD-MSCs elevated cell viability and attenuated cell apoptosis after co-culture with myocardial cells (19). To further clarify the regulatory role of TIPE2 in allogeneic heart transplantation, this study performed heart transplantation in mice. By injecting TIPE2-overexpressed hAD-MSCs into the caudal vein, the regulation and mechanisms of immune rejection were studied after heart transplantation. We present the following article in accordance with the ARRIVE reporting checklist (available at https://dx.doi.org/10.21037/jtd-21-1034). 


\section{Methods}

\section{Animals}

A total of 40 4-week-old male BALB/c (B6, H-2 ${ }^{\mathrm{b}}$, recipient) and C57BL/6 (H-2 ${ }^{\mathrm{d}}$, donor) mice weighing 20-30 g were provided by SPF (Beijing) Biotechnology Co., Ltd. for surgeries. All mice met the animal feeding conditions of being specific pathogen free. Both donors and recipients were fasted and were forbidden to drink before the procedures. All animal experiments were performed under a project license (No. 2020120101) according to the Guide for the Care and Use of Laboratory Animals by the Zunyi Medical University Ethics Committee. A protocol was prepared before the study without registration.

\section{bAD-MSCs}

Isolation, culture, identification, and enrichment of hADMSCs, along with TIPE2 gene modification of hAD-MSCs, were described in our previous article (16).

\section{Anesthesia}

Mice underwent anesthesia with the U.S. MATRX inhalation anesthesia machine for small animals. By adding sevoflurane for inhalation, the mice were first induced in an anesthesia state in an induction box. Next, they were taken out and supplied with oxygen through masks at $0.3-0.6 \mathrm{~L} / \mathrm{min}$ and sevoflurane at $2-3 \%$, which ensured that the mice were under anesthesia during the entire procedure. Regular observations were made including heart rate, respiration, pain response, and muscle tension to monitor and adjust the depth of anesthesia. After administration, the appropriate anesthesia effect was achieved when the pain response in the skin disappeared, and signs of muscle relaxation and smooth breathing were indicated with a heart rate of roughly 100 times/min and 40 breaths/min.

\section{Heart transplantation procedures}

The heart transplantation process is shown in Figure 1.

\section{Recipient}

The mouse was fixed after anesthesia, with cervical skin prepared and disinfected. The skin was incised at the line between the right mandibular angle and the right midpoint of the clavicle. The right external jugular vein was freed $1-1.5 \mathrm{~cm}$ to the distal end away from the clavicle. The right sternocleidomastoid muscle was severed and isolated, and the right common carotid artery was exposed and dissociated at a distance of $1-1.5 \mathrm{~cm}$. Lengths of arteries and veins were essential to successful transplantation and great effort was paid to eliminate fat and connective tissues adjacent to veins. The proximal end of the external jugular vein was closed with a clamp, then the distal end was ligated and disconnected. A cannula was inserted into the dissociated end of the external jugular vein and fixed at the groove with a 10-0 microsuture. A common carotid artery cannula was prepared following the same method. The arteriovenous cannulas were washed with $125 \mathrm{U} / \mathrm{mL}$ heparin saline to avoid thrombosis. Then, wet gauze immersed in normal saline was applied to cover them.

\section{Donor}

Initially, the abdominal midline was excised and the surgical field was fully exposed. Then, $1-2 \mathrm{~mL} 125 \mathrm{U}$ heparin saline was injected into the inferior vena cava. The abdominal aorta and inferior vena cava were cut off after 2-3 min of systemic heparinization for bloodletting. The thorax was cut through the axillary front, the anterior chest wall was removed, and the superior vena cava was cut to prevent neck reflux. The donor's heart was rinsed with $125 \mathrm{U}$ heparin normal saline by injection from the inferior septum through the inferior vena cava until the color of the donor's heart turned from pale to pink. The peripheral tissue of the heart was cleaned with cotton swabs, then the bilateral thymus was removed. A 5-0 ligation suture went through the transverse sinus of the pericardium and the left and right auricles, and the superior and inferior vena cava and pulmonary veins were ligated back together. The main pulmonary artery was freed and cut off at a distance of $2-3 \mathrm{~mm}$ including the ligation bundle. Finally, the donor's heart was collected.

\section{Transplantation}

Wet gauze immersed in normal saline was cushioned for the incision edge of the recipient. The donor's heart was placed invertedly into the cervical part of the recipient mouse. Next, the end of the cardiopulmonary artery of the donated heart was inserted into the recipient vein cannula. A loop was tied at another groove with a $10-0$ microsuture. The arterial end cannula was connected with the donor aorta in the same method. First, the vein was loosened and then followed the artery at a slow pace. Just then, it was visible that the donor's heart was filling, starting from the aorta, coronary artery, and pulmonary artery in turn, with colors 

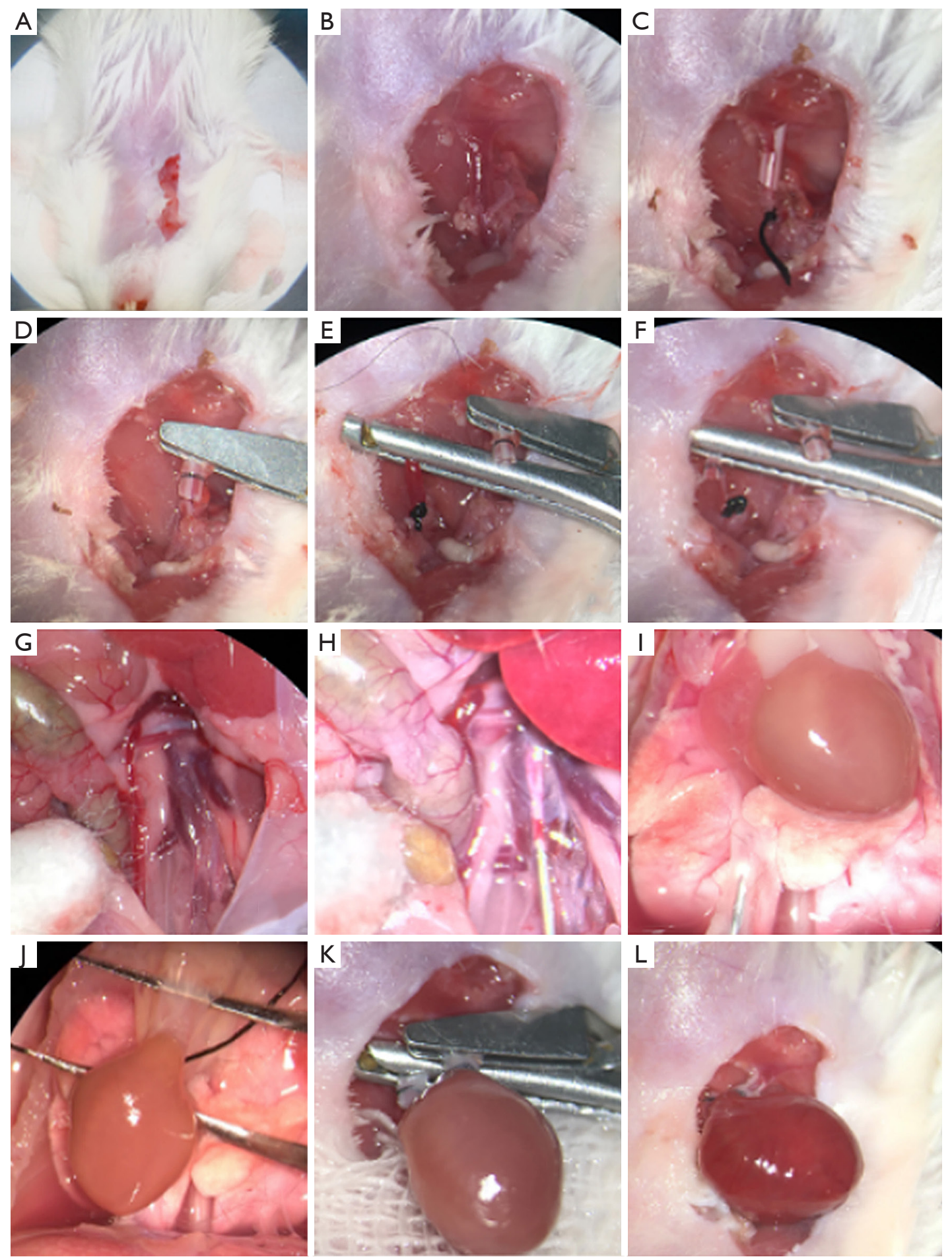

Figure 1 The heart transplantation process. (A) A mouse was laid supine and fixed with a cut in the neck. (B) The right external jugular vein was freed. (C) The vein was cut off and the cannula was passed through. (D) Vein was externalized and fixed. (E) The right common carotid artery was freed. (F) Artery was externalized and fixed. (G) The inferior vena cava was exposed at the level of the abdominal kidney of the donor. (H) Heparin was injected by the inferior cavity. (I) The donor heart was rinsed from the inferior septum and inferior cavity. (J) The main pulmonary artery of the donor heart was freed. (K) Arteriovenous anastomosis. (L) Transplantation completed, clamps were loosened and heat beat was resumed. 
turning into bright red. The donor's heart resumed beating soon. Positions of both the donor heart and the cannula were adjusted before being fixed. Forming angles and distortion were avoided for both the artery and vein. Finally, the incision of the recipient's neck was sutured. Once the surgery was completed, the recipient was administered an intramuscular injection of $0.5 \mathrm{~mL}$ normal saline, then placed in a constant temperature chamber and fed in a single cage. Mostly, the mouse was able to turn over, crawl, drink water, and eat on its own after 15-20 min. The food supplied was not too hard.

\section{Calculation of the spleen index and thymus index of mice}

After cervical heart transplantation, mice were fed in a solitary cage. The conditions of the mice were observed and recorded daily. Subsequently, the mice were sacrificed on the 7 th day after the surgery and weighed. Both the spleen and thymus were obtained and weighed by electronic scales. The spleen index and thymus index were calculated as follows: viscera index $=$ viscera mass $(\mathrm{mg}) /$ body mass $(\mathrm{g})$.

\section{Pathological sections of transplanted heart and hematoxylin and eosin (HE) staining}

On the 7 th day after the surgery, the mice were anesthetized and heart specimens were collected from the cervical part. Blood stains were rinsed with cold normal saline, and then the specimens were immersed in a fixing solution containing $4 \%$ paraformaldehyde for $24 \mathrm{~h}$.

Paraffin-embedded tissue blocks were cut into $4 \mu \mathrm{m}$ sections and each was baked at $70{ }^{\circ} \mathrm{C}$ for $2 \mathrm{~h}$ prior to deparaffinization. Conventional deparaffinization and hydration were performed, and sections were rinsed with distilled water for $5 \mathrm{~min}$. Sections underwent HE staining for $5 \mathrm{~min}$, rinsed with running water for $3 \mathrm{~s}$, differentiated in $1 \%$ hydrochloric acid ethanol for $2 \mathrm{~s}$, then rinsed with running water for $30 \mathrm{~s}$ and distilled water for $2 \mathrm{~s}$. Then, $0.5 \%$ eosin staining was performed for $3 \mathrm{~min}$, then sections were rinsed with distilled water for $2 \mathrm{~s}$ again, followed by dehydration and transparency. Slides were fixed with a neutral gum seal and observed under a microscope (Olympus, Japan) then imaged.

\section{Flow cytometry}

Lymphocytes of the spleen and heart were extracted. The spleen was placed in a petri dish and thoroughly ground on a $200 \mu \mathrm{m}$ strainer. The heart was injected with $3 \mathrm{~mL}$ type IV collagenase and cut into pieces, then digested for $30 \mathrm{~min}$ at $37^{\circ} \mathrm{C}$. Then, $2 \%$ fetal bovine serum (FBS) in phosphatebuffered saline (PBS) was added and the mixture was ground with a $3 \mathrm{~mL}$ syringe, then filtered with a $100 \mu \mathrm{m}$ strainer. The cells were transferred into a $50 \mathrm{~mL}$ centrifuge tube, with $50 \mathrm{~mL}$ of $2 \%$ FBS in PBS, and centrifuged at $400 \mathrm{~g}$ for $5 \mathrm{~min}$. Next, $5 \mathrm{~mL}$ of red blood cell (RBC) lysis solution was added at RT for $5 \mathrm{~min}$. The lysis buffer was diluted with $5 \mathrm{~mL} \mathrm{2 \%} \mathrm{FBS} \mathrm{in} \mathrm{PBS,} \mathrm{and} 20 \mathrm{~mL}$ PBS was added then centrifuged $\left(400 \mathrm{~g}, 5 \mathrm{~min}, 4^{\circ} \mathrm{C}\right)$. After repeat filtration, $25-30 \mathrm{~mL}$ PBS was added, then centrifuged again $\left(400 \mathrm{~g}, 10 \mathrm{~min}\right.$ at $\left.4{ }^{\circ} \mathrm{C}\right)$. After centrifugation, the cells were resuspended in $10 \mathrm{~mL}$ cell staining buffer (CSB). The cells were counted and approximately 5 million spleen lymphocytes and graft lymphocytes were transferred into a $12 \times 75 \mathrm{~mm}$ flow tube, which was then centrifuged at $400 \mathrm{~g}$ for 5 min at $4{ }^{\circ} \mathrm{C}$.

Cell staining: anti-CD16/32 in $50-100 \mu \mathrm{L}$ CSB was added (antibody concentration 1:50). Surface staining antibody and $50-100 \mu \mathrm{L}$ CSB were added $(100 \mu \mathrm{L}$ added in graft cells). After gentle shaking, cells were stained at $4{ }^{\circ} \mathrm{C}$ in the dark for $30 \mathrm{~min}$, then washed with CSB. Cells were then centrifuged at $400 \mathrm{~g}$ for $5 \mathrm{~min}$ at $4{ }^{\circ} \mathrm{C}$ and washed twice. Then, a $200 \mu \mathrm{L}$ fixing solution was added for surface staining and simple intracellular staining, following by rinsing with $2 \% \mathrm{FBS}$ in PBS. Cells were centrifuged at $600 \mathrm{~g}$ for $5 \mathrm{~min}$ at $4{ }^{\circ} \mathrm{C}$ and washed twice, then $1 \mathrm{~mL}$ fix/ perm buffer was added and mixed quickly. Membranes were ruptured for $60 \mathrm{~min}$ at $4{ }^{\circ} \mathrm{C}$. The intracellular staining antibody was added for staining and shaken gently. Cells were placed in a shaker avoiding light for $60 \mathrm{~min}$. After rinsing, the cells were resuspended for the loading test.

\section{Real-time PCR (RT-PCR)}

Total RNA was isolated from TIPE2-transfected or empty vector-transfected hAD-MSCs with TRIzol reagent (Invitrogen, USA) as per the manufacturer's instructions. The first-strand cDNA was generated with gDNA Eraser (Takara, Japan) by the PrimeScript ${ }^{\mathrm{TM}} \mathrm{RT}$ reagent kit. Quantitative RT-PCR (qRT-PCR) assays were carried out by loading SYBR Green PCR Kits (Takara, Japan) in the real-time PCR system (Bio-Rad, USA). The reactions were made following the instructions of the manufacturer. By adopting the $2^{-\Delta \Delta C T}$ method, the relative expression levels were calculated. Following the quantification of cardiomyocyte target expressions, the relevant levels were 
normalized to $\beta$-actin. Primers were listed in Table S1.

\section{Western blot}

Total proteins were extracted from TIPE2-transfected or empty vector-transfected hAD-MSCs in ice-cold lysis buffer and BCA kits (Takara, Japan) were applied subsequently for quantification. A mixture of $5 \times$ SDS loading buffer was prepared with $500 \mu \mathrm{g}$ protein at a 4:1 ratio, which was collected from the total protein of the samples. The mixed proteins, approximately $6 \mu \mathrm{g} / \mu \mathrm{L}$, were denatured in boiling water for $5 \mathrm{~min}$. Following separation at $60 \mu \mathrm{g} / \mathrm{lane}$ via SDSPAGE gels (Jinruisi, China), the proteins were delivered to polyvinylidene difluoride (PVDF) membranes (Millipore, USA). Blocked by $5 \%$ fat-free milk powder as required, the membranes were subsequently cultured supplemented with primary antibodies overnight at $4{ }^{\circ} \mathrm{C}$. The employed primary antibodies were listed as below: anti-p38 (Biyuntian, AF7668, 1:1,000), anti-phospho-p38 (anti-p-p38) (Beyotime, AF5884, 1:1,000), anti-ERK (Beyotime, AF1315, 1:1,000), anti-phospho-ERK (anti-p-ERK) (Beyotime, AF1891, 1:1,000), anti-IL-10 (Abcam, ab34843, 1:1,000), anti-TGF- $\beta$ (Beyotime, AF0297, 1:1,000), anti-phospho-TGF- $\beta$ (anti-pTGF- $\beta$ ) (Beyotime, AF5863, 1:1,000), anti-IFN- $\gamma$ (Abcam, ab77246, 1:1,000) and anti- $\beta$-actin (Abcam, ab8227, 1:1,000). Following three cycles of washing using TBST (5 min each), the membranes were incubated for $1.5-2 \mathrm{~h}$ at room temperature by addition of secondary antibodies (Beyotime, China) and rinsed 3 times with TBST. An ECL detection system (Thermo Fisher Scientific, USA) was utilized for observing the expression levels of proteins, which were quantified subject to the Image J software (NIH, USA) and normalized to $\beta$-actin.

\section{Statistical analysis}

All of the experimental data were expressed as mean and standard deviation. Pairwise comparison of the collected data was subjected to $t$-tests as per post hoc Tukey's test using SPSS 23.0 (SPSS, Inc., USA) for significant analysis. The values of $\mathrm{P}$ obtained less than 0.05 were regarded as statistically significant.

\section{Results}

\section{Evaluation of heart transplantation procedures}

Recipients received an intramuscular injection of $0.5 \mathrm{~mL}$ of normal saline after the heart surgery. Palpations after the procedure demonstrated that the transplanted heart was beating. Strong pulses were visible in the neck and a regular rhythm was observed. Three palpations were performed daily. Strong pulse and regular rhythm were displayed at roughly 140-160 beats/min. Over the several days after the successful transplantation, the heart beat faster and faster, reaching 300-400 beats/min. In cases where the transplanted heart was obviously smaller or enlarged with a weak pulse, hardened presentation, and slow heart rhythm by palpation, it was likely that complications occurred including arterial thrombosis, arterial compression, and stenosis of the venous anastomotic orifice, which revealed a failure of the procedure. The recipient mice were palpated daily in the neck in the morning, at noon, and in the evening. Successful surgery was defined as the transplanted heart beating well and lasting over 72 hours. The anastomotic time of heart transplantation lasted $11.3 \pm 3.5$ minutes in this study, while the ischemia time of the donor's heart was $16.6 \pm 3.8$ minutes. The total operation was $64.6 \pm 5.8$ minutes. Luckily, 24 mice survived and only 1 died after transplantation, with a survival rate of $96 \%$. The cervical heart transplantation model in mice constructed in this study was stable and reliable, both in terms of efficiency and survival rate.

\section{TIPE2 extended the survival time of mice after transplantation}

Survival time differences in mice were observed by tail intravenous injection of both hAD-MSCs (control group) and TIPE2-hAD-MSCs (experimental group). The median survival time was 9 days in the control group $(n=12)$ and 19.5 days in the experimental group $(\mathrm{n}=12)$. Statistical analysis of data of the two groups was performed (Figure 2A). Conclusions from the study suggested that the survival time of the TIPE2 group was significantly longer than that of the control group after transplantation.

\section{Detection of the distribution and organ indexes of bAD-MSCs in mice}

On the 5th day after hAD-MSC transplantation, in vivo imaging of mice in each group demonstrated no red fluorescence expression in the entire body of the mice, both in the control group and the blank group. Furthermore, hAD-MSC expression with a strong DiR red fluorescent marker was observed in the caudal part of mice among 
A

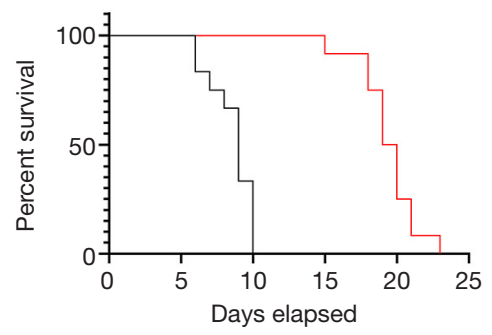

C

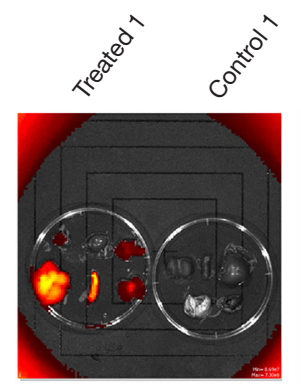

+ Control

+ Treated
B

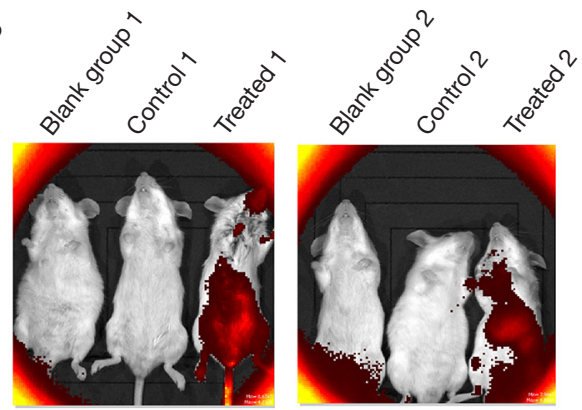

$\mathrm{D}$

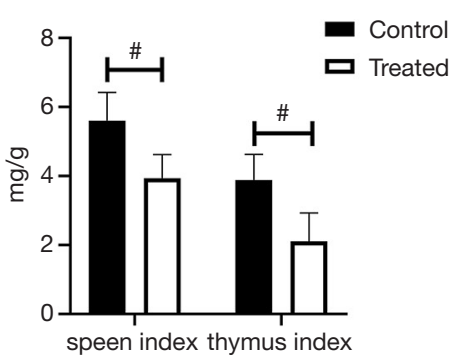

Figure 2 Detection of the distribution and organ indexes of hAD-MSCs in mice. (A) Survival time analysis between the two groups was conducted using GraphpadPrism 6.0 software. (B,C) A small animal in vivo imaging system detected the fluorescence distribution. (D) Detection of the organ indexes. ", $\mathrm{P}<0.05$. hAD-MSCs, human amnion-derived mesenchymal stem cells.

the test groups. Red fluorescence was visible on the body surface of the projected positions of both the liver and spleen. A low level of fluorescence was visible in the lungs and the neck (Figure 2B).

The mice were sacrificed before organs including hearts (both the donor hearts and the mouse autologous hearts), livers, spleens, lungs, and kidneys were removed to capture images. No red fluorescence expression was displayed in the viscera of the control group, while obvious red fluorescence expression was observed in the treatment group. Stronger signals were visible in the liver, spleen, and lungs, followed by the kidneys. A minor amount of expression was visualized in 1 donor heart (Figure 2C).

As shown in Figure 2D, the spleen and thymus indexes were, respectively, $3.94 \pm 0.68$ and $2.11 \pm 0.82 \mathrm{mg} / \mathrm{g}$ for the TIPE2 treatment group, and $5.61 \pm 0.82$ and $3.89 \pm 0.74 \mathrm{mg} / \mathrm{g}$ for the control group. The results suggested that both the thymus and spleen indexes of recipient mice were significantly reduced in the TIPE2 treatment group.

\section{TIPE2 effectively protected the myocardium}

Myocardial fiber fracture was observed by HE staining in the control group. Neutrophils, lymphocytes, and tissue cells were visualized in the myocardial interstitium, as well as some moderate fibroblasts. Cardiac fibers were irregular, accompanied by muscle fiber edema and disappearing continuity. Rhabdomyolysis was visible and intracardiac myocardial coagulation necrosis was revealed. In contrast, the myocardial fibers were neat and continuous in the TIPE2 treatment group, with a smaller quantity of inflammatory cells found in the myocardial tissues. The results showed that the injection of TIPE2-hAD-MSCs significantly reduced the infiltration of intramyocardial lymphocytes from the transplanted hearts in mice with ectopic heart transplantation. Meanwhile, it protected normal structures of the grafted heart and maintained normal functions of the heart, and greatly inhibited the acute rejection of the transplanted heart, thereby effectively protecting cardiac tissues (Figure $3 A$ ).

\section{TIPE2 reduced the number of lymphocytes in the recipient beart and spleen}

According to flow cytometry analysis of the grafted hearts, the number of lymphocytes expressed by $\mathrm{CD} 8^{+} \mathrm{T}$ cells 
A

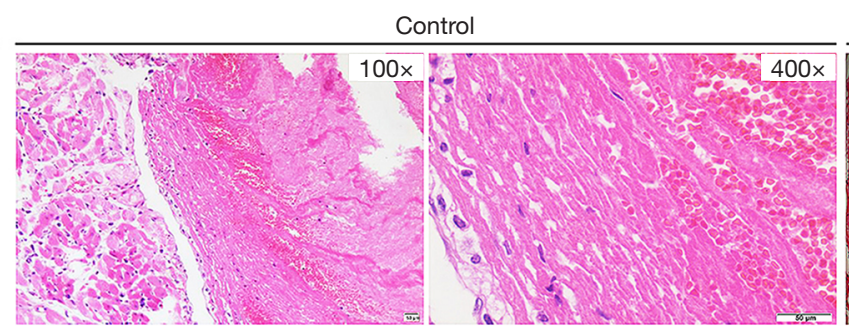

B

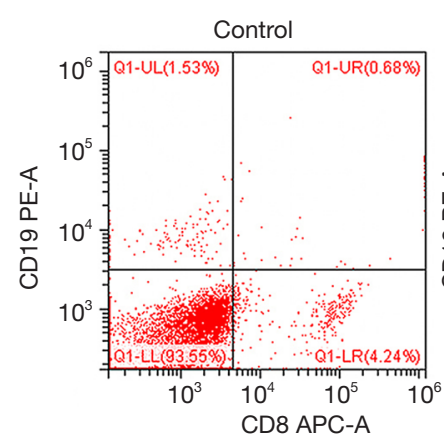

CD19+

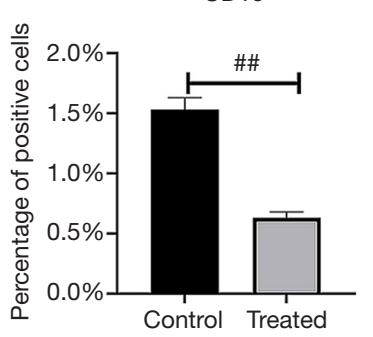

D

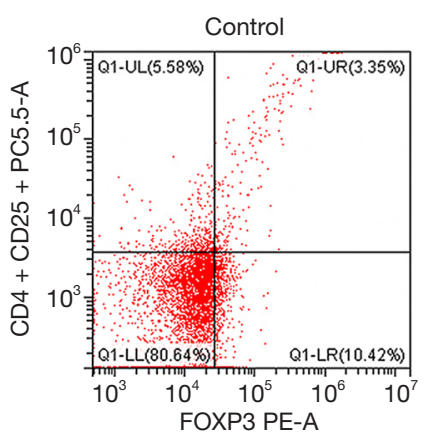

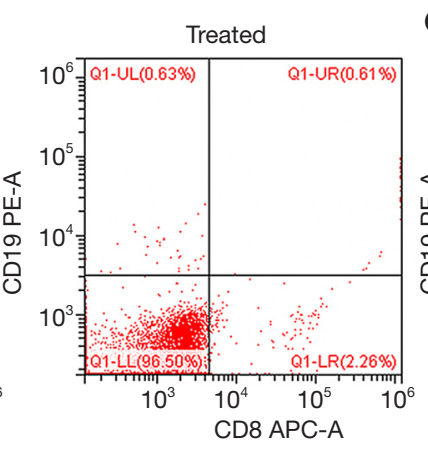

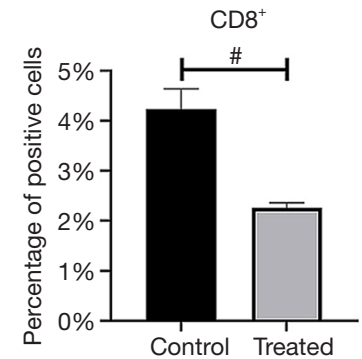

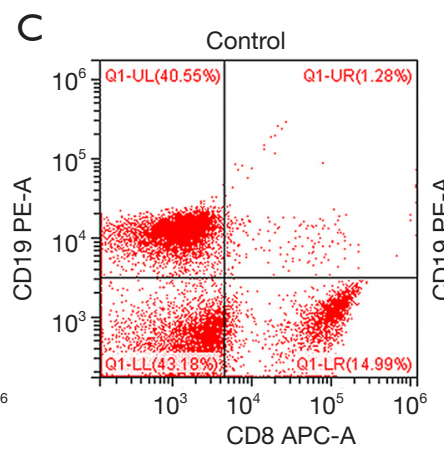

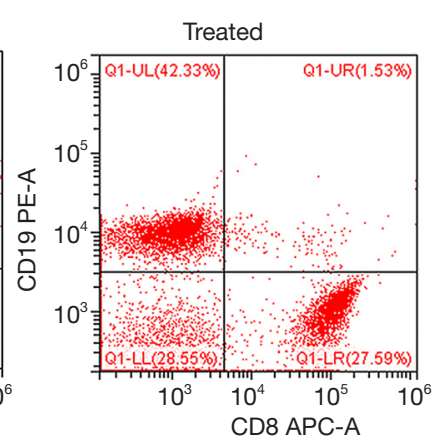

$\mathrm{CD}^{+}$
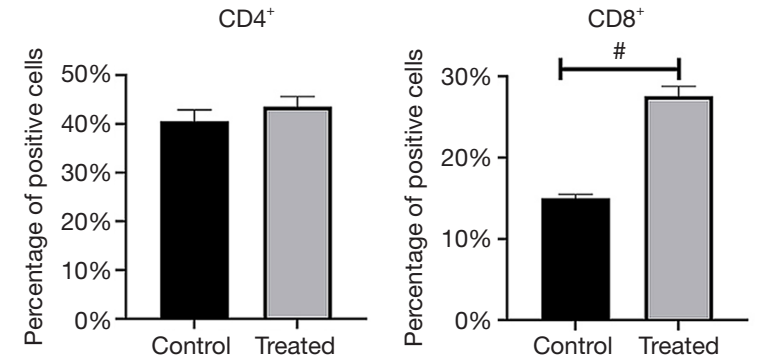

Treated

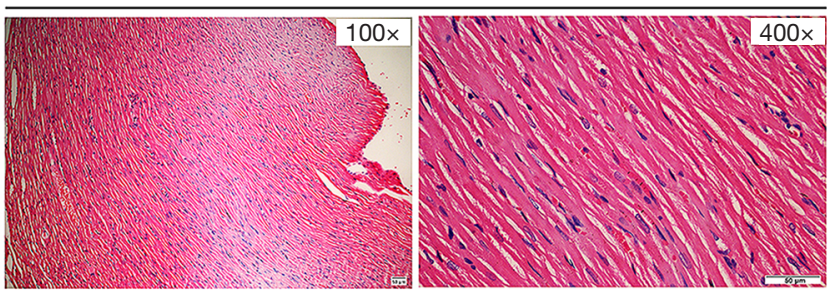

E
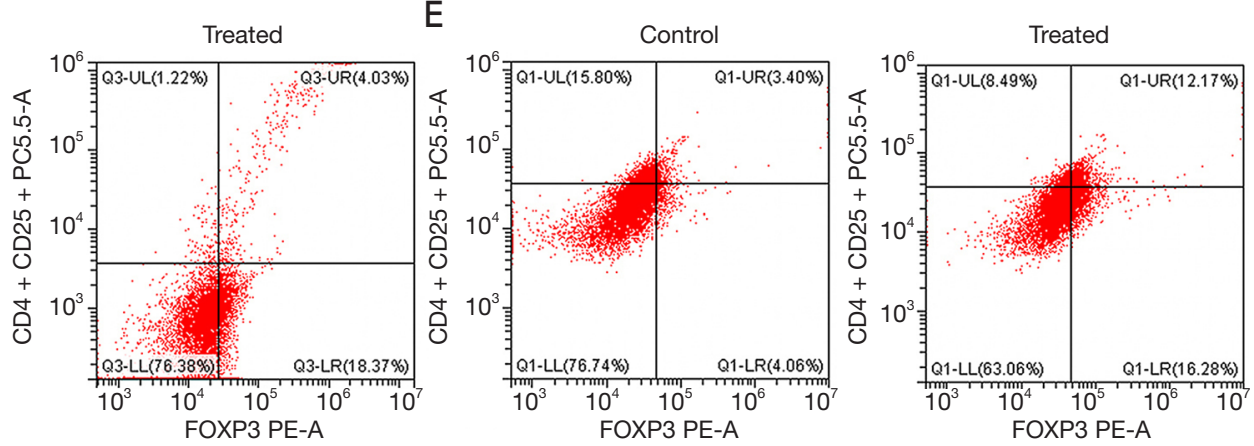
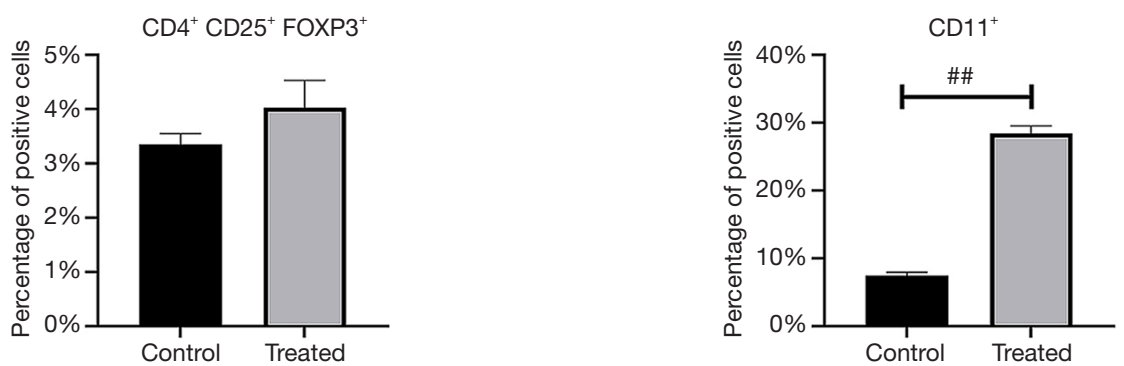

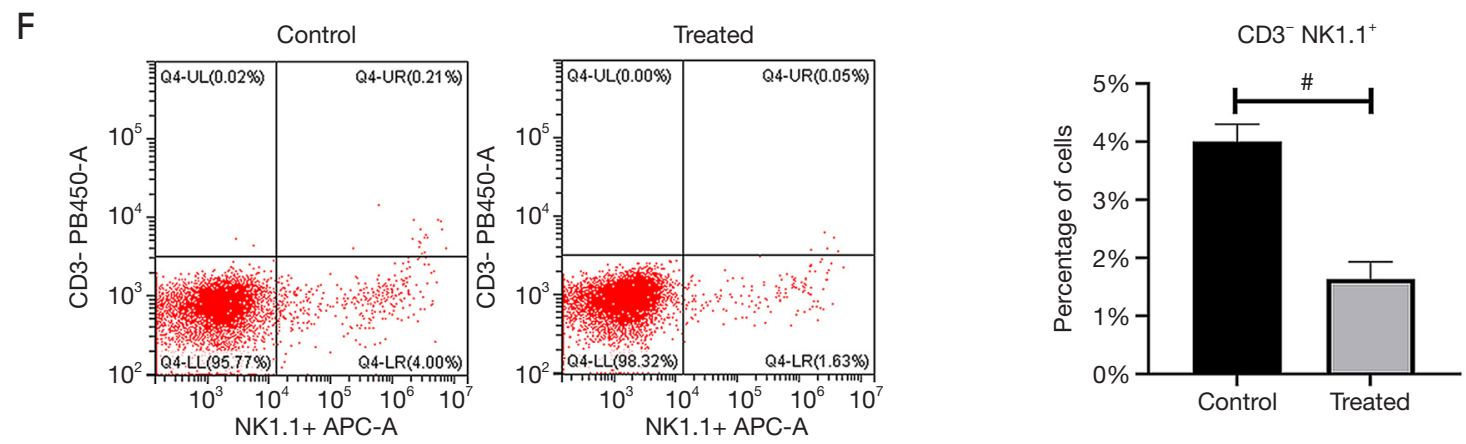

Figure 3 The expression of TIPE2 on myocardial and immune cells was detected by HE staining and flow cytometry. (A) HE staining. Scale bar $=50 \mu \mathrm{m}$. (B) The percentages of $\mathrm{T}$ and B cells in the heart were measured by flow cytometry. (C-F) The percentages of T cells, B cells, and NK cells in the spleen were detected by flow cytometry. ${ }^{*}, \mathrm{P}<0.05 ;{ }^{* \#}, \mathrm{P}<0.01$. TIPE2, tumor necrosis factor- $\alpha$-induced protein-8 like-2; $\mathrm{HE}$, hematoxylin and eosin.

and $\mathrm{CD} 19^{+} \mathrm{B}$ cells was remarkably lower in TIPE2 group than that of control group, indicating that TIPE2 greatly attenuated the number of both $\mathrm{CD} 8^{+} \mathrm{T}$ cells and $\mathrm{CD} 19^{+}$ $\mathrm{B}$ cells of graft myocardium in mice with ectopic heart transplantation (Figure 3B).

Based on the flow cytometry analysis of the recipient's spleens, the number of $\mathrm{CD} 8^{+} \mathrm{T}$ cells in the recipient's spleens was remarkably reduced in the TIPE2 group compared with the control group, while there was no significant difference in $\mathrm{CD}^{+} \mathrm{T}$ cells. Such evidence confirmed that TIPE2 minimized the number of $\mathrm{CD} 8^{+} \mathrm{T}$ cells in the recipient's spleen (Figure 3C). The number of Treg $\left(\mathrm{CD} 4^{+} \mathrm{CD} 25^{+} \mathrm{FOXP}^{+}\right)$cells in the recipient's spleens was notably higher in the TIPE2 group than in the control group, which substantiated that TIPE2 significantly elevated the number of Treg cells in the recipient spleen (Figure 3D). The number of CD $11^{+}$B lymphocytes was obviously higher in the recipient spleens of the TIPE2 group than the control group, which demonstrated that TIPE2 significantly elevated the number of $\mathrm{CD} 11^{+} \mathrm{B}$ lymphocytes in the recipient spleen (Figure $3 E$ ). The number of $\mathrm{CD}^{-} \mathrm{NK} 1.1^{+}$ NK cells in the recipient spleens of the TIPE2 group was greatly reduced compared to the control group, suggesting that TIPE2 largely reduced the number of NK cells in the recipient spleen (Figure $3 F$ ).

\section{Detection of the expression levels of immune response-related factors}

To further detect the immune tolerance regulation mechanisms of TIPE2 after heart transplantation, the expression levels of $I F N-\gamma, I L-10, T G F-\beta, E R K$, and $p 38$ genes in these experimental groups were determined using qRT-PCR and western blot assays. Results from the detection showed that the mRNA expression levels of $p 38$, $E R K$, and $I F N-\gamma$ were reduced in the TIPE2-hAD-MSCs group compared with the control group (Figure 4A-4E). Reduction of the protein expression and decrease of the phosphorylation levels were exhibited in the TIPE2-hADMSCs group (Figure 4F). Conversely, by overexpressing TIPE2, expression levels of TGF- $\beta$ and $I L-10$ were elevated, which indicated that TIPE2 mediated the regulation of the expression of cellular immune response factors.

\section{Discussion}

Currently, the first and most effective clinical option is organ transplantation for patients with end-stage organ failure. However, acute and chronic graft rejection associated with the graft and the recipient is a key factor affecting the long-term survival of the recipient. It is recognized that transplant rejection is mediated primarily by cellular immunity. In recent years, Tregs and Bregs have been identified as major negative immune regulators of cellular damage $(20,21)$. Considerable attention has been paid by researchers to the roles in autoimmune diseases, tumor immunity, and transplantation immunity. However, the mechanisms still require further investigations. The previous study of this project at the cellular level revealed that the overexpression of TIPE2 in hAD-MSCs significantly enhanced the ability of the cells in terms of both immune tolerance and anti-inflammation (19). Further exploration at the animal level was conducted in this study which aimed to identify the therapeutic effect 


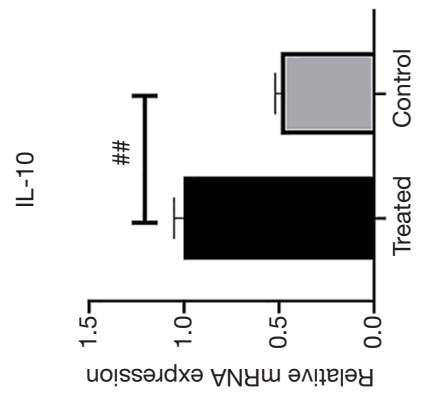

Ш

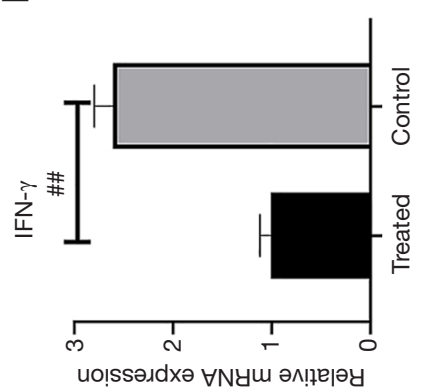

口

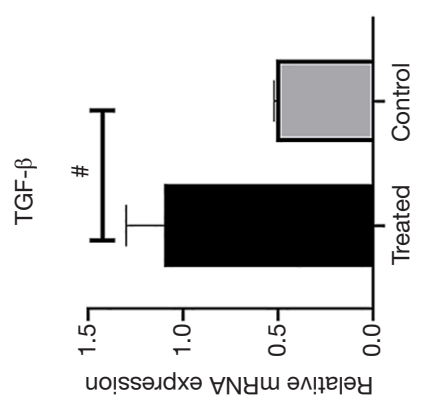

$\cup$

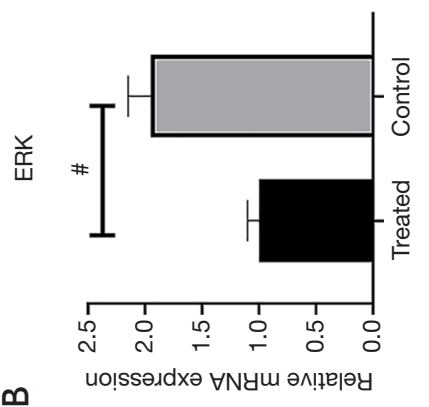

$\infty$

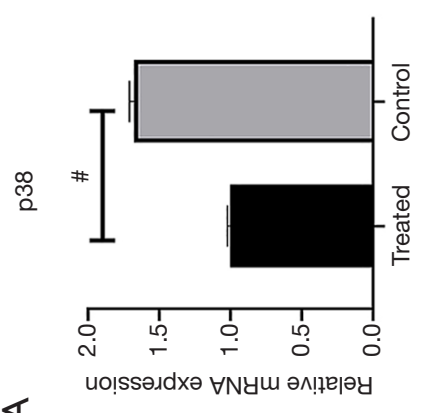

$\varangle$
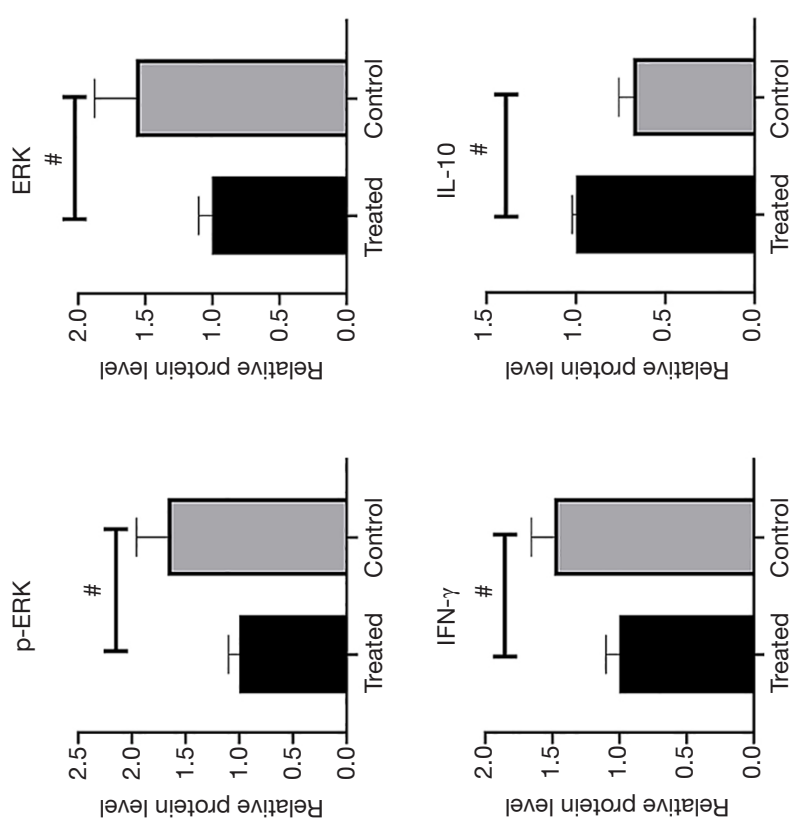

है
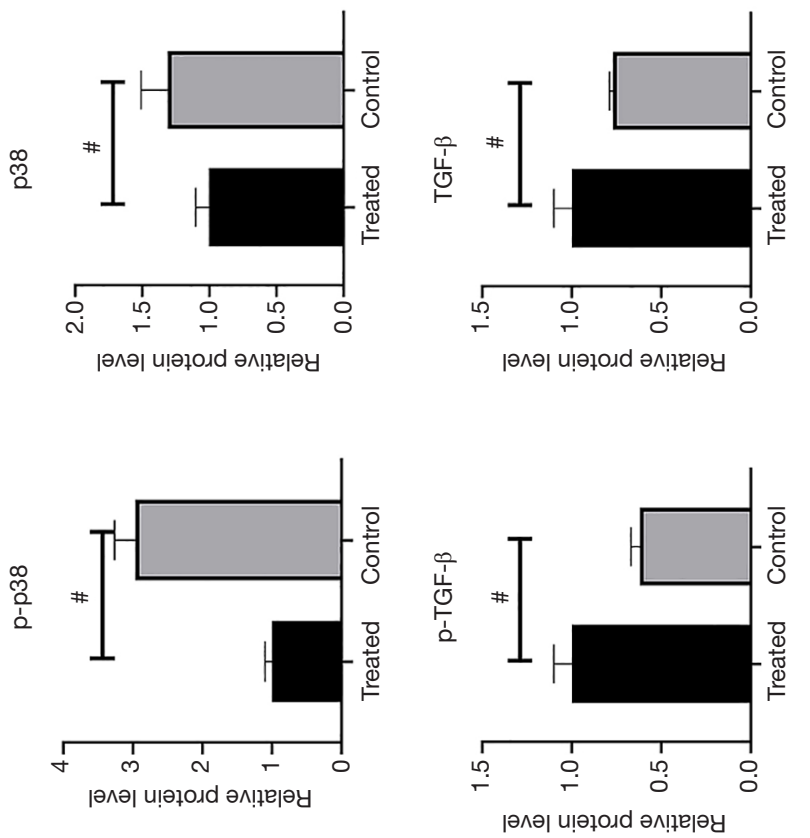

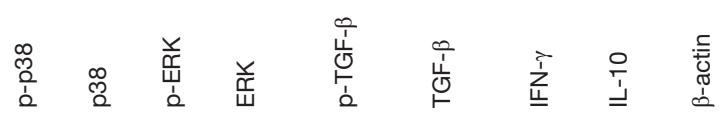

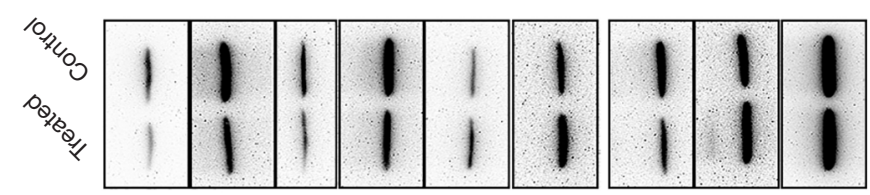

น

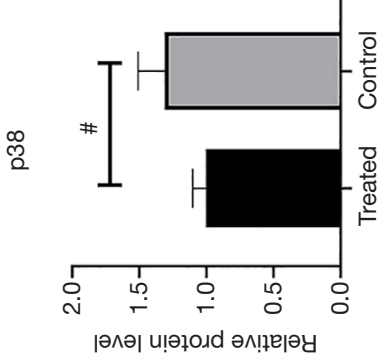

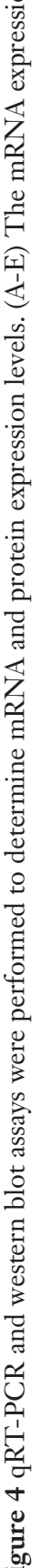

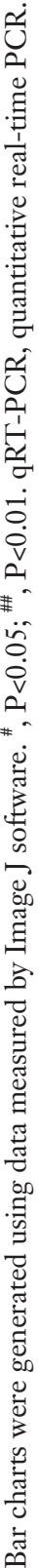


of TIPE2 on immune rejection after heart transplantation by constructing a heart transplantation model. The results attested that after allogeneic heart transplantation in mice, caudal vein injection of hAD-MSCs overexpressed with TIPE2 could effectively protect myocardial tissue structures, minimize the spleen and thymus indexes of the recipient mice, and attenuate the content of lymphocytes in the heart, thereby remarkably improving the survival time and survival rates of the recipient mice.

It has been reported that TIPE2 mediates the signal transduction of both $\mathrm{T}$ cell receptors (TCRs) and toll-like receptors (TLRs), achieving negative regulation of inherent and adaptive immune responses, thereby maintaining immune homeostasis (22). By directly binding to caspase-8, TIPE2 could also promote cell apoptosis via inhibiting $A P-1$ and $N F-\kappa B$. Moreover, TIPE2 played a significant role in regulating the function of Treg cells when expressed on Treg cells $(23,24)$. By silencing the TIPE2 gene, the expression levels of FoxP3 and CTLA-4 were minimized on the surface of Treg cells, thereby attenuating the immunosuppressive effect of Treg cells (25). The expression levels of $I L-2, I L-4$, and $I F N-\gamma$ of TIPE2-/- mice were significantly elevated. Conversely, the expression level of $I L-10$ was significantly reduced, which proved that TIPE2 worked as an important negative regulatory protein in the immune system $(25,26)$. Furthermore, this study verified the diverse effects of TIPE2 at the animal level in improving immune tolerance, increasing the expression of $I L-10$, and reducing the expression of $I F N-\gamma$.

Be it the total number or types of current organ transplantation, China has taken the lead in the clinical field. However, there is still a long way to go to catch up with the world's advanced level in fundamental research, especially in immune rejection. The success of stable induction of immune tolerance plays a critical role in the breakthrough of organ transplantation. After heart transplantation, the graft is subject to both immune rejection and ischemiareperfusion injury in the recipient. Not only does the duration of ischemia have an important impact on graft survival, but the ischemia-reperfusion injury itself increases cardiac tissue immunogenicity. As of now, few studies have been carried out on the therapeutic approaches which have resisted immune rejection and prevented or minimized reperfusion injury simultaneously. However, TIPE2 gene-modified hAD-MSC transplantation was proven to significantly contribute to both immune regulation and the alleviation of ischemia-reperfusion injury. The results of this research confirmed that the injection of TIPE2- modified hAD-MSCs could significantly improve the heart tissue structures of recipient mice, thereby increasing the survival rates of mice. This study provides conclusive evidence for the efficacy of TIPE2-modified hAD-MSCs in the treatment of cardiac transplantation diseases, offering theoretical support and an experimental basis for future research in developing new approaches to genetically modified stem cell therapies.

\section{Conclusions}

In vivo experiment results in this study demonstrated that TIPE2 gene-modified hAD-MSCs could decrease the lymphocyte content, induce immune tolerance, and improve the efficacy of allogeneic heart transplantation.

\section{Acknowledgments}

Funding: This study was funded by the National Natural Science Foundation of China (\#8166020277, \#81960051).

\section{Footnote}

Reporting Checklist: The authors have completed the ARRIVE reporting checklist. Available at https://dx.doi. org/10.21037/jtd-21-1034

Data Sharing Statement: Available at https://dx.doi. org/10.21037/jtd-21-1034

Conflicts of Interest: All authors have completed the ICMJE uniform disclosure form (available at https://dx.doi. org/10.21037/jtd-21-1034). The authors have no conflicts of interest to declare.

Ethical Statement: The authors are accountable for all aspects of the work in ensuring that questions related to the accuracy or integrity of any part of the work are appropriately investigated and resolved. This study was approved by the Zunyi Medical University Ethics Committee (No. 2020120101) and performed according to the Guide for the Care and Use of Laboratory Animals by the Zunyi Medical University Ethics Committee. A protocol was prepared before the study without registration.

Open Access Statement: This is an Open Access article distributed in accordance with the Creative Commons Attribution-NonCommercial-NoDerivs 4.0 International 
License (CC BY-NC-ND 4.0), which permits the noncommercial replication and distribution of the article with the strict proviso that no changes or edits are made and the original work is properly cited (including links to both the formal publication through the relevant DOI and the license). See: https://creativecommons.org/licenses/by-nc-nd/4.0/.

\section{References}

1. Bao Z, Li J, Zhang P, et al. Toll-like receptor 3 activator preconditioning enhances modulatory function of adipose-derived mesenchymal stem cells in a fully MHC-mismatched murine model of heterotopic heart transplantation. Ann Transplant 2020;25:e921287.

2. Weiss ARR, Lee O, Eggenhofer E, et al. Differential effects of heat-inactivated, secretome-deficient MSC and metabolically active MSC in sepsis and allogenic heart transplantation. Stem Cells 2020;38:797-807.

3. Hua D, Ju Z, Gan X, et al. Human amniotic mesenchymal stromal cells alleviate acute liver injury by inhibiting the pro-inflammatory response of liver resident macrophage through autophagy. Ann Transl Med 2019;7:392.

4. Wu SM, Zhang WX, Wang MH, et al. Proteomic analysis of the immunosuppressive effects of mesenchymal stem cells in a rat heart transplantation model. Adv Clin Exp Med 2013;22:785-94.

5. Eggenhofer E, Renner P, Soeder Y, et al. Features of synergism between mesenchymal stem cells and immunosuppressive drugs in a murine heart transplantation model. Transpl Immunol 2011;25:141-7.

6. Zhou H, Jin Z, Liu J, et al. Mesenchymal stem cells might be used to induce tolerance in heart transplantation. Med Hypotheses 2008;70:785-7.

7. Stamatopoulos A, Stamatopoulos T, Gamie Z, et al. Mesenchymal stromal cells for bone sarcoma treatment: Roadmap to clinical practice. J Bone Oncol 2019;16:100231.

8. Özdemir AT, Özgül Özdemir RB, Kırmaz C, et al. The paracrine immunomodulatory interactions between the human dental pulp derived mesenchymal stem cells and CD4 T cell subsets. Cell Immunol 2016;310:108-15.

9. English K, Barry FP, Field-Corbett CP, et al. IFN-gamma and TNF-alpha differentially regulate immunomodulation by murine mesenchymal stem cells. Immunol Lett 2007;110:91-100.

10. Wang D, Huang S, Yuan X, et al. The regulation of the Treg/Th17 balance by mesenchymal stem cells in human systemic lupus erythematosus. Cell Mol Immunol
2017;14:423-31.

11. Díaz-Coránguez M, Segovia J, López-Ornelas A, et al. Transmigration of neural stem cells across the blood brain barrier induced by glioma cells. PLoS One 2013;8:e60655.

12. Hosseini SM, Sani M, Haider KH, et al. Concomitant use of mesenchymal stem cells and neural stem cells for treatment of spinal cord injury: a combo cell therapy approach. Neurosci Lett 2018;668:138-46.

13. Hakim R, Covacu R, Zachariadis V, et al. Mesenchymal stem cells transplanted into spinal cord injury adopt immune cell-like characteristics. Stem Cell Res Ther 2019;10:115.

14. Gorjipour F, Hosseini-Gohari L, Alizadeh Ghavidel A, et al. Mesenchymal stem cells from human amniotic membrane differentiate into cardiomyocytes and endothelial-like cells without improving cardiac function after surgical administration in rat model of chronic heart failure. J Cardiovasc Thorac Res 2019;11:35-42.

15. Wang $\mathrm{Q}$, Wei C, Ma L, et al. Inflammatory cytokine $\mathrm{TNF}-\alpha$ promotes corneal endothelium apoptosis via upregulating TIPE2 transcription during corneal graft rejection. Graefes Arch Clin Exp Ophthalmol 2018;256:709-15.

16. Zhao Y, Wang Y, Zhu MS, et al. Expression pattern of tumor necrosis factor- $\alpha$-induced protein 8 -like 2 in acute rejection of cardiac allograft. Transplant Proc 2018;50:293-8.

17. Luan YY, Yao RQ, Tong S, et al. Effect of tumor necrosis factor- $\alpha$ induced protein 8 like- 2 on immune function of dendritic cells in mice following acute insults. Oncotarget 2016;7:30178-92.

18. Huang H, Cui $Y$, Tian $Z$, et al. Tumor necrosis factor- $\alpha-$ induced protein 8 -like 2 downregulation reduces $\mathrm{CD}^{+} \mathrm{T}$ lymphocyte apoptosis in mice with thermal injury. Med Sci Monit 2019;25:7547-56.

19. Wang F, Pan S, Yao G, et al. TIPE2 Improves the immune tolerance of human amniotic mesenchymal stem cells. All Life 2020;13:233-43.

20. Liu Z, Gu J, Qin Z, et al. Decreased Foxp3 and function of Tregs caused immune imbalance and liver injury in patients with autoimmune liver diseases post-liver transplantation. Ann Transl Med 2020;8:534.

21. Song J, Du G, Chen W, et al. The advantage of Sirolimus in amplifying regulatory B cells and regulatory $\mathrm{T}$ cells in liver transplant patients. Eur J Pharmacol 2020;869:172872.

22. Sun H, Gong S, Carmody RJ, et al. TIPE2, a negative regulator of innate and adaptive immunity that maintains 
immune homeostasis. Cell 2008;133:415-26.

23. Ji J, Zhang YY, Fan YC. TIPE2 as a potential therapeutic target in chronic viral hepatitis. Expert Opin Ther Targets 2019;23:485-93.

24. Zhao P, Wang S, Jiang J, et al. TIPE2 sensitizes osteosarcoma cells to cis-platin by down-regulating MDR1 via the TAK1 - NF- $\mathrm{B}$ and - AP-1 pathways. Mol Immunol 2018;101:471-8.

25. Luan YY, Yao YM, Zhang L, et al. Expression of tumor

Cite this article as: Wang F, Yao G, Pan S, Mao X, Zhao X, Li C, Hong Z, Liang G, Yu L, Hu X, Peng W. TIPE2-modified human amnion-derived mesenchymal stem cells promote the efficacy of allogeneic heart transplantation through inducing immune tolerance. J Thorac Dis 2021;13(8):5064-5076. doi: $10.21037 /$ jtd-21-1034 necrosis factor- $\alpha$ induced protein 8 like- 2 contributes to the immunosuppressive property of CD4(+)CD25(+) regulatory T cells in mice. Mol Immunol 2011;49:219-26.

26. Liu MW, Su MX, Zhang W, et al. Rhodiola rosea suppresses thymus T-lymphocyte apoptosis by downregulating tumor necrosis factor- $\alpha$-induced protein 8-like-2 in septic rats. Int J Mol Med 2015;36:386-98.

(English Language Editor: C. Betlazar-Maseh) 
Supplementary

Table S1 Primer sequences of real-time RT-PCR

\begin{tabular}{lll}
\hline Genes & Direction & Sequence (5'-3') \\
\hline$p 38$ & Forward & GGCTCCTGAGATCATGCTGAACTG \\
& Reverse & AGTCAACAGCTCGGCCATTATGC \\
ERK & Forward & GAAAGCATTACCTTGACCAG \\
& Reverse & CTTGGAGTCAGCATTGG \\
TGF- $\beta$ & Forward & CCCTATATTTGGAGCCTGGA \\
& Reverse & GTTGGTTGTAGAGGGCAAGG \\
INF- $\gamma$ & Forward & TGTCATCGATCGCACCTGA \\
& Reverse & TGTGCTGGATCTGTGGGTTG \\
& Forward & TGCCTAACATGCTTCGAGATCTCCG \\
$\beta$-actin & Reverse & TTAGAGGGAGGTCAGGGAAACAGC \\
& Forward & CGATGGGTTGTACCTTGTCTAC3 \\
\hline
\end{tabular}

RT-PCR, reverse transcription PCR. 